\title{
Tau lepton physics at Belle
}

\section{Hisaki Hayashii*i}

Nara Women's University, Japan

E-mail: hayashii@hepl.phys.nara-wu.ac.jp

We present recent results on the high statistics study of the decay $\tau^{-} \rightarrow \pi^{-} \pi^{0} v_{\tau}$ and the precise measurement of $\tau$-mass, obtained using data recorded by the Belle detector at the $\mathrm{KEKB} e^{+} e^{-}$ collider at the center-of-mass energy of $10.6 \mathrm{GeV}$.

The branching fraction for $\tau^{-} \rightarrow \pi^{-} \pi^{0} v_{\tau}$ and the invariant mass spectrum of the resulting $\pi^{-} \pi^{0}$ system are measured using $72.2 \mathrm{fb}^{-1}$ of data. The branching fraction obtained is $(25.15 \pm 0.04 \pm 0.31) \%$, where the first error is statistical and the second is systematic. The unfolded $\pi^{-} \pi^{0}$ mass spectrum is used to determine resonance parameters for the $\rho(770), \rho^{\prime}(1450)$, and $\rho^{\prime \prime}(1700)$ mesons. We also use this spectrum to estimate the hadronic contribution to the anomalous magnetic moment of the muon.

The mass of the $\tau$-lepton has been measured in the decay modes $\tau \rightarrow 3 \pi v_{\tau}$ and $\tau \rightarrow 3 \pi \pi^{0} v_{\tau}$ using a pseudomass technique. The result is $1776.71 \pm 0.25$ (stat) \pm 0.62 (syst) MeV. The upper limit on the relative mass difference between positive and negative $\tau$ lepton $\mid\left(M_{\tau^{+}}-M_{\tau^{-}}\right) / M_{\text {average }}$ is $5.0 \times 10^{-4}$ at $90 \%$ C.L. The mass difference result is one order of magnitude lower than the previous limit.

International Europhysics Conference on High Energy Physics

July 21 st - 27th 2005

Lisboa, Portugal

*Speaker.

†n behalf of the Belle Collaboration. 


\section{High Statistics Study of the Decay $\tau \rightarrow \pi^{-} \pi^{0} v_{\tau}[1]$}

Among the decay channels of the $\tau$ lepton, the decay $\tau \rightarrow \pi^{-} \pi^{0} v_{\tau}$ has the largest branching fraction. The decay is dominated by intermediate resonances and thus provides information on the properties of the $\rho$ (770), $\rho^{\prime}(1450)$, and $\rho^{\prime \prime}(1700)$ mesons and their interference. Since leptons do not participate in the strong interaction, hadronic $\tau$ decays provide a clean environment for studying the dynamics of hadronic states in an interesting energy range dominated by resonances. Under the Conserved Vector Current (CVC) theorem, the $\pi^{-} \pi^{0}$ mass spectrum in this range can be used to improve the theoretical error on the anomalous magnetic moment of the muon $a_{\mu}=\left(g_{\mu}-2\right) / 2$. Recent calculations of $a_{\mu}$ indicate that there is a significant difference between $e^{+} e^{-}$-based and $\tau$-based calculations in particular in the $2 \pi$ mode [2]. To clarify the situation, new measurements are important. We report here a measurement of the branching fraction and the mass spectrum for $\tau^{-} \rightarrow \pi^{-} \pi^{0} v_{\tau}$ using $72.2 \mathrm{fb}^{-1}$ data collected by the Belle detector [3]. This sample is 50 times larger than that of previous studies.

The branching fraction for $\tau^{-} \rightarrow h^{-} \pi^{0} v_{\tau}\left(\mathscr{B}_{h \pi^{0}}\right)$ is determined by dividing the signal yield $N_{h \pi^{0}}$ by the number of selected generic $\tau^{+} \tau^{-}$pairs $N_{\tau \tau}$ :

$$
\mathscr{B}_{h \pi^{0}}=\frac{N_{h \pi^{0}}}{2 N_{\tau \tau}} \cdot \frac{\left(1-b^{\text {feed-down }}-b^{\text {non- } \tau}\right)}{\left(1-b_{\tau \tau}\right)} \cdot\left(\frac{\varepsilon_{\tau \tau}}{\varepsilon_{h \pi^{0}}^{\tau}}\right) \cdot \frac{1}{\varepsilon_{h \pi^{0}}^{\mathrm{ID}}} .
$$

In this formula, $b_{\tau \tau}$ is the background fraction in the $\tau^{+} \tau^{-}$sample, $\varepsilon_{\tau \tau}$ is the efficiency of the $\tau$-pair selection, $\varepsilon_{h \pi^{0}}^{\tau}$ is the efficiency for $\tau^{-} \rightarrow h^{-} \pi^{0} v$ decays to pass the $\tau$-pair selection, and $\varepsilon_{h \pi^{0}}^{I D}$ is the efficiency for $\tau^{-} \rightarrow h^{-} \pi^{0} v$ decays satisfying the $\tau$-pair selection to pass the $h^{-} \pi^{0}$ selection. The parameters $b^{\text {feed-down }}$ and $b^{\text {non- } \tau}$ are the fraction of $h^{-} \pi^{0} \nu$ candidates coming from other $\tau$ decay modes and from non- $\tau$ processes, respectively. Several common uncertainties cancel in this ratio. $22.7 \times 10^{6}$ generic $\tau$-pairs are selected from $72.2 \mathrm{fb}^{-1}$ data by applying loose criteria consisting of low multiplicity and existence of the missing particles. Within the $\tau$-pair sample, $\tau^{-} \rightarrow h^{-} \pi^{0} v_{\tau}$ decays are reconstructed by requiring that there be both one charged track and one $\pi^{0}$ in one hemisphere. Resulting $5.55 \times 10^{6} \tau^{-} \rightarrow h^{-} \pi^{0} v_{\tau}$ decays are selected with a good signal-tobackground ratio. Here $h^{-}$denotes $\pi$ or $K$. The efficiencies and the background fractions relevant in this experiment are $\varepsilon_{\tau \tau} / \varepsilon_{h \pi^{0}}^{\tau}=1.112 \pm 0.003, \varepsilon_{h \pi^{0}}^{\mathrm{ID}}=42.62 \pm 0.13 \%, b_{\tau \tau}=7.66 \pm \pm 0.03 \%$, $b_{h \pi^{0}}^{\text {feed }- \text { down }}=5.98 \pm 0.07 \%, b_{h \pi^{0}}^{\text {non }-\tau}=2.45 \pm 0.06 \%$. Inserting all values in Eq.(1.1) gives

$$
\mathscr{B}_{h \pi^{0}}=(25.60 \pm 0.04 \pm 0.31) \%,
$$

where the first error is statistical and the second is systematic. Subtracting the small kaon-channel branching fraction listed in the PDG [4] [ $\mathscr{B}_{K^{-}} \pi^{0}=(0.45 \pm 0.03) \%$ ] gives a $\tau \rightarrow \pi^{-} \pi^{0} v_{\tau}$ branching fraction of

$$
\mathscr{B}_{\pi \pi^{0}}=(25.15 \pm 0.04 \pm 0.31) \% .
$$

The branching fraction is measured with $1.2 \%$ accuracy which is better than that in the previous experiments (except for the ALEPH result).

Figure 1 shows the unfolded $\pi^{-} \pi^{0}$ mass squared ( $s=M_{\left(\pi \pi^{0} \text { unf. }\right)}^{2}$ ) spectrum $d N / d s$ in the decay $\tau^{-} \rightarrow \pi^{-} \pi^{0} v_{\tau}$. The unfolding is carried out by using the singular-value-decomposition(SVD) 


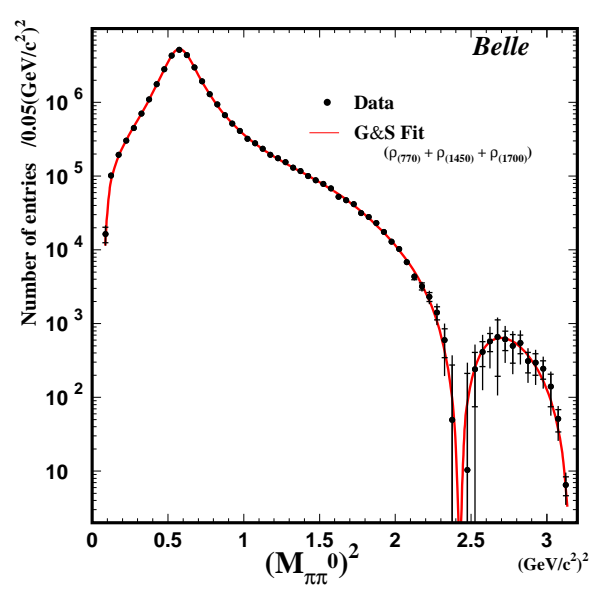

Figure 1: Fully-corrected $M_{\pi \pi^{0}}^{2}$ distribution for $\tau^{-} \rightarrow \pi^{-} \pi^{0} v_{\tau}$. The solid curve is the result of a fit to the Gounaris-Sakurai model with $\rho(770), \rho^{\prime}(1450)$, and $\rho^{\prime \prime}(1700)$ resonances. All parameters are floated.

\begin{tabular}{l|c|c}
\hline \hline Parameter & $\begin{array}{c}\text { Fit result } \\
\text { (all free) }\end{array}$ & $\begin{array}{c}\text { Fit result } \\
\text { (fixed } \phi_{\gamma} \text { ) }\end{array}$ \\
\hline \hline$M_{\rho}\left(\mathrm{MeV} / \mathrm{c}^{2}\right)$ & $774.6 \pm 0.2 \pm 0.3$ & $774.3 \pm 0.2 \pm 0.3$ \\
$\Gamma_{\rho}(\mathrm{MeV})$ & $150.6 \pm 0.3 \pm 0.5$ & $150.0 \pm 0.3 \pm 0.5$ \\
$M_{\rho^{\prime}}\left(\mathrm{MeV} / \mathrm{c}^{2}\right)$ & $1336 \pm 12 \pm 23$ & $1436 \pm 15 \pm 23$ \\
$\Gamma_{\rho^{\prime}}(\mathrm{MeV})$ & $471 \pm 29 \pm 21$ & $553 \pm 31 \pm 21$ \\
$|\beta|$ & $0.090 \pm 0.009 \pm 0.013$ & $0.161 \pm 0.020 \pm 0.013$ \\
$\phi_{\beta}($ degree $)$ & $123.7 \pm 5.0 \pm 7.0$ & $149.1 \pm 2.4 \pm 7.0$ \\
$M_{\rho^{\prime \prime}}\left(\mathrm{MeV} / \mathrm{c}^{2}\right)$ & $1600 \pm 13 \pm 4$ & $1804 \pm 16 \pm 4$ \\
$\Gamma_{\rho^{\prime \prime}}(\mathrm{MeV})$ & $255 \pm 19 \pm 79$ & $567 \pm 81 \pm 79$ \\
$|\gamma|$ & $0.062 \pm 0.015 \pm 0.015$ & $0.136 \pm 0.024 \pm 0.015$ \\
$\phi_{\gamma}($ degree $)$ & $-64.1 \pm 7.9$ & {$[0]$} \\
\hline$\chi^{2} /$ d.o.f & $55 / 51$ & $94 / 52$ \\
\hline \hline
\end{tabular}

Table 1: Results of fitting the $M_{\pi \pi^{0}}^{2}$ distribution for $\tau^{-} \rightarrow \pi^{-} \pi^{0} v_{\tau}$ to the Gounaris-Sakurai model with $\rho(770), \rho^{\prime}(1450)$, and $\rho^{\prime \prime}(1700)$ resonances. The results for two cases, all parameters floated (the second column) and fixed $\phi_{\gamma}$ (the third column) are shown. For both cases, the first error is statistical and the second one is systematic.

method given in Ref. [5]. In the spectrum, the $\rho$ peak and the shoulder due to the $\rho^{\prime}(1450)$ are clearly visible. The solid line is a fit with three Breit-Wigner functions $(B W)$, including the $\rho$, $\rho^{\prime}(1450)$, and $\rho^{\prime \prime}(1700)$ resonances. The pion form factor in the fitting formula is given by

$$
F_{\pi}(s)=\frac{1}{1+\beta+\gamma}\left(B W_{\rho}+\beta \cdot B W_{\rho^{\prime}}+\gamma \cdot B W_{\rho^{\prime \prime}}\right),
$$

where the parameters $\beta$ and $\gamma$ (denoting the relative size of the two resonances) are in general complex. For the Breit-Wigner shape, the Gounaris-Sakurai(GS) model [6] is used. 
The results of the fit are summarized in Table 1 for two cases, all parameters are floated and fixed $\phi_{\gamma}$. The $\chi^{2}$ becomes worse if $\phi_{\gamma}$ is fixed to zero. In addition to $\rho(770)$ and $\rho^{\prime}(1450)$ mesons, the production of the $\rho^{\prime \prime}(1700)$ in $\tau^{-}$decays has been unambiguously demonstrated and its parameters determined.

The unfolded spectrum is used to evaluate the $2 \pi$ contribution to the muon anomalous magnetic moment $a_{\mu}^{\pi \pi}$. Our results after integrating over the region $\sqrt{s}=0.50-1.80 \mathrm{GeV} / \mathrm{c}^{2}$ is

$$
\left.a_{\mu}^{\pi \pi}[0.50,1.80]=(462.6 \pm 0.6 \text { (stat. }) \pm 3.2 \text { (sys.) } \pm 2.3(\text { isospin })\right) \times 10^{-10},
$$

where the first error is statistical, the second is systematic, and the third arises from isospin violation. Note that this result includes a correction for the isospin breaking effects $\delta a_{\mu}^{\pi \pi}=$ $(-1.8 \pm 2.3) \times 10^{-10}$, which comes from the $\rho-\omega$ interference effect and the mass difference of $\pi^{-}$and $\pi^{0}$. More detailed description of the analysis is available in Ref.[1].

\section{Measurement of mass of the $\tau$-lepton [7]}

The present PDG value of the $\tau$ mass [4] is dominated by the result of the BES Collaboration[8] and has an accuracy about $0.3 \mathrm{MeV}$. The high statistics of the Belle data allows a measurement with the same level of accuracy.

Analysis presented here is based on the $253 \mathrm{fb}^{-1}$ data taken with the Belle detector at the KEKB asymmetric-energy $e^{+} e^{-}$collider. In the analysis, so-called pseudomass $M_{\min }$ defined by

$$
M_{\text {min }}=\sqrt{M_{\mathrm{X}}^{2}+2\left(E_{\text {beam }}-E_{\mathrm{X}}\right)\left(E_{\mathrm{X}}-P_{\mathrm{X}}\right)},
$$

is used as an estimator of the $\tau$ mass. Here $M_{\mathrm{X}}, E_{\mathrm{X}}$ and $P_{\mathrm{X}}$ are the invariant mass, energy and absolute value of the momentum of the hadronic system in a hadronic $\tau$ decay $\tau^{-} \rightarrow X^{-} v_{\tau}$, respectively. Since $M_{\min }$ should be less than or equal to the $\tau$ lepton mass, one can use the threshold position obtained from the fit to the experimental $M_{\min }$ distribution as an estimator of the $\tau$ mass. The initial and final state radiation as well as the finite momentum resolution of the detector smear the shape of the edge for $M_{\min }$ near the $\tau$ mass. The contributions from background processes have smooth behavior near the $\tau$ mass. A possible correction caused by the method itslef and all these effects is studied by Monte-Carlo and the correction is equal to $\mathbf{M}_{\tau}^{\text {obs }}-\mathbf{M}_{\tau}^{\text {true }}=0.70 \pm 0.40 \mathrm{MeV}$.

The distribution of $M_{\min }$ for the selected $\tau \rightarrow 3 \pi v_{\tau}$ data is shown in Fig.2. The line is the result of the fit. After applying the correction mentioned above, the result for the $\tau$ mass is

$$
M_{\tau}=1776.71 \pm 0.25 \text { (stat) } \pm 0.62 \text { (syst) } \mathrm{MeV}
$$

where the first error is statistical and the second is systematic. For the systematic uncertainty, following contributions are considered:

1) A sample of the $\Upsilon(1 s) \rightarrow \mu^{+} \mu^{-}$decay, measured as the decay products of $\Upsilon(2 S, 3 S)$ to $\Upsilon(1 S) \pi^{+} \pi^{-}$is used to determine the absolute value of the momentum scale in the tracking system. The uncertainty of the tracking system gives the systematic error of $0.39 \mathrm{MeV}$. 2) The variation of the fit range and threshold function gives the systematic uncertainty of $0.40 \mathrm{MeV}$. 3) The beam energy is known with the accuracy better than $1.5 \mathrm{MeV}$. This uncertainty provides the uncertainty of $0.26 \mathrm{MeV}$. 4) Systematic uncertainty coming from the misidentified $\tau$ decays is negligible. 


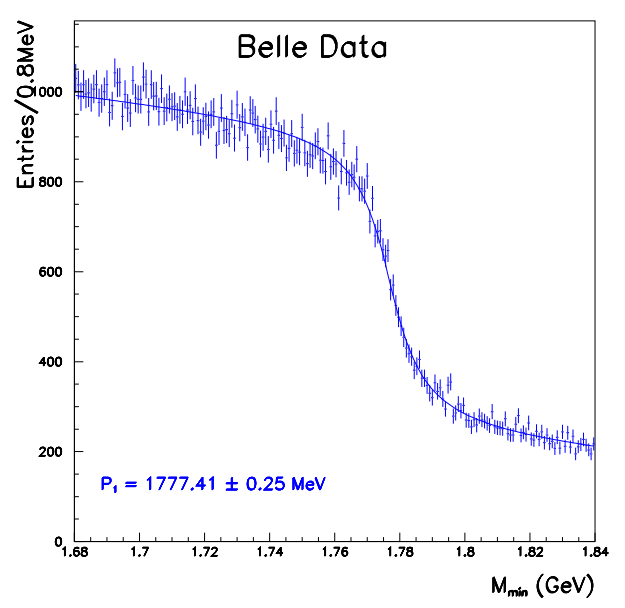

Figure 2: Pseudomass distribution $M_{\min }$ for $\tau \rightarrow 3 \pi^{ \pm} v_{\tau}$ decays. The points with error bars are data and the solid line is the result of the fit.

\subsection{CPT Test}

The pseudomass method allows a separate measurement of the masses of the positively and negatively charged $\tau$ leptons. The mass difference obtained from the independent fits to the $M_{\min }$ distribution is $M_{\tau^{+}}-M_{\tau^{-}}=-0.12 \pm 0.45 \mathrm{MeV}$, consistent with no difference between a positively and negatively charged $\tau$ lepton. By taking into account the possible systematic shift in the measurement of particle and antipartcile momenta in the tracking system, we obtained an upper limit on the relative mass difference to be $\left|\left(M_{\tau^{+}}-M_{\tau^{-}}\right)\right| / M_{\text {average }}<5.0 \times 10^{-4}$ at $90 \%$ C.L. This limit is one order of magnitude lower than the previous result obtained by the OPAL experiment[9].

\section{References}

[1] Belle Collaboration, K. Abe et al., in preparation.

[2] A recent review is given in M. Passera, J. Phys. G 31, R75 (2005).

[3] Belle Collaboration, A. Abashian et al., Nucl. Instr. and Meth. A 479, 117 (2002).

[4] Review of Particle Physics, S. Eidelman, et al., Phys. Lett. B 592, 1 (2004).

[5] A. Höcker and V. Kartvelishvili, Nucl. Instr. Meth. A 372, 469 (1996).

[6] G.J. Gounaris and J.J. Sakurai, Phys. Rev. Lett. 21, 244 (1968).

[7] Belle Collaboration, K. Abe et al., hep-ex/0511038.

[8] BES Collaboration, J.Z Bai, et al., Phys. Rev. D 53, 20 (1996).

[9] OPAL Collaboration, G. Abbiendy, et al., Phys. Lett. B 492, 23 (2000). 